\title{
Anabases
}

ANABASES Traditions et réceptions de l'Antiquité

6 | 2007

Varia

\section{Le musée de l'Arles et de la Provence antiques met Ingres à l'honneur}

Adeline Grand-Clément

\section{(2) OpenEdition}

1 Journals

Édition électronique

URL : http://journals.openedition.org/anabases/3392

DOI : 10.4000/anabases.3392

ISSN : 2256-9421

Éditeur

E.R.A.S.M.E.

Édition imprimée

Date de publication : 1 octobre 2007

Pagination : 242-248

ISSN : 1774-4296

\section{Référence électronique}

Adeline Grand-Clément, " Le musée de l'Arles et de la Provence antiques met Ingres à l'honneur »,

Anabases [En ligne], 6 | 2007, mis en ligne le 01 janvier 2012, consulté le 14 novembre 2019. URL

http://journals.openedition.org/anabases/3392 ; DOI : 10.4000/anabases.3392

Ce document a été généré automatiquement le 14 novembre 2019.

(c) Anabases 


\title{
Le musée de l'Arles et de la Provence antiques met Ingres à l'honneur
}

\author{
Adeline Grand-Clément
}

1 Jean-Auguste-Dominique Ingres (1780-1867), lorsqu'il dirige l'Académie de France à Rome, prône auprès de ses élèves un rapport à l'art antique qui s'écarte du dogmatisme du néo-classicisme : « il est nécessaire d'étudier les antiques et les maîtres, non pour les imiter, mais [...] pour apprendre à voir ». Le peintre ne considère plus les œuvres du passé comme des modèles inaccessibles et indépassables, mais bien comme des outils de la connaissance, dont l'artiste moderne doit se saisir afin d'élaborer son propre travail de créateur. L'heure n'est plus à l'attitude contemplative et à l'admiration béate, mais au regard aiguisé et à l'observation méthodique.

2 Les choix effectués par les musées, dans la façon de présenter au public leurs collections, ont souvent oscillé entre ces deux modes de rapport à l'Antique, entre la mise en scène esthétique et la présentation didactique. Au Musée de l'Arles et de la Provence antiques (MAPA), qui renferme les vestiges archéologiques de la ville et de son territoire, depuis le néolithique jusqu'à la fin de l'antiquité tardive, c'est l'objectif pédagogique qui prime. Les remarquables efforts réalisés en matière de muséographie en particulier le recours à des maquettes confectionnées par des scientifiques - et l'attention soutenue portée à l'accueil des différents publics en témoignent. On y vient pour « apprendre à voir » et comprendre le patrimoine antique de la région. Le lieu était donc tout trouvé pour abriter une exposition consacrée à Ingres et au rôle déterminant joué par la référence antique dans la genèse de son œuvre.

3 Fabrice Denise retrace l'histoire de l'établissement et de ses collections, avant de nous expliquer les missions dévolues au Service des Publics, dont il a la charge. 


\section{L'Histoire du Musée de l'Arles et de la Provence antiques}

4 Depuis le $\mathrm{XvI}^{\mathrm{e}}$ siècle jusqu'à la Révolution, des amateurs arlésiens éclairés recueillent, collectionnent et parfois même publient les antiquités qui proviennent du sol de leur cité et qui font leur fierté. Nombre de religieux les imitent, à commencer par les archevêques en leur palais. Mais l'ensemble le plus éloquent est celui réuni par les consuls de la ville et exposé à l'Hôtel de Ville à partir de 1614. D'autres lieux d'expositions ouvrent également. Ainsi, au XviII ${ }^{e}$ siècle, chez les religieuses de la Miséricorde, un jardin public d'antiquités propose autour des deux colonnes du théâtre, encore debout, un choix d'œuvres provenant de ce lieu.

5 Le premier musée public d'antiquités ouvert à tous, l'un des plus anciens de France, est créé aux Alyscamps, à l'instigation du père Dumont, en 1784. Une convention lie les frères Minimes du couvent de Saint-Honorat à la ville. Elle autorise les religieux à « rassembler et réunir dans un seul et même endroit les divers monuments d'antiquités, qui se trouvaient épars dans la ville ou son terrain " pour leur permettre "d'être visités le plus aisément par les curieux». En 1785, des objets jusque là conservés à l'Hôtel de Ville sont transportés en ce nouveau lieu. La Révolution française entraîne le pillage du couvent des Minimes; de nombreuses œuvres sont irrémédiablement endommagées. La plupart de celles qui restent sont rapatriées dans l'Hôtel de Ville. Les collections connaissent alors d'autres vicissitudes. En 1801, le préfet Delacroix veut faire main basse sur ces richesses afin de les présenter à Marseille. Il faut alors toute l'habileté de Pierre Véran, qui devient ensuite le premier conservateur du musée d'Arles, pour éviter le transfert et empêcher le départ des plus belles pièces pour le Louvre. Il parvient à convaincre les autorités et la ville de créer un musée à Arles. Par un décret impérial du 19 nivôse an XIII (9 janvier 1805), l'église Sainte-Anne est dévolue à la conservation des antiquités.

Les découvertes dues aux travaux et au dégagement des monuments, effectués au cours des décennies suivantes, enrichissent les collections du musée d'une façon considérable. La place vient à manquer et, en 1935, Fernand Benoît décide d'installer les œuvres chrétiennes dans l'ancienne église du Collège des Jésuites. Cette première division des collections ne suffit pas, car, suite aux effets de la guerre, beaucoup d'antiquités provenant de Trinquetaille ou des cryptoportiques sont mises au jour, ce qui provoque un nouvel engorgement du musée. Pour résoudre le problème, on choisit en 1954 de ne plus y présenter que le lapidaire. Tout le reste prend le chemin des réserves, au grand dam des visiteurs et des Arlésiens.

7 En 1968, à l'occasion d'un congrès international à Mexico, Jean-Maurice Rouquette, alors conservateur en chef des musées d'Arles, expose la nécessité de regrouper en un lieu unique toutes les collections archéologiques de la ville, afin de les présenter dans leur chronologie et d'une manière pédagogique et attractive. Le site retenu pour l'implantation du futur musée est la presqu'île formée par le Rhône et le canal d'Arles à Port-de-Bouc, qui se trouve en dehors de l'enceinte historique de la ville. Il offre l'avantage de servir de lien entre deux quartiers d'Arles (la ville ancienne et le quartier de Barriol) et surtout de mettre en valeur le Cirque romain, un hippodrome de 400 mètres de long sur plus de 100 mètres de large, qui pouvait accueillir jusqu'à 20000 personnes ${ }^{1}$. En 1984, le projet de l'architecte Henri Ciriani, adepte du mouvement moderne, est retenu. Celui-ci promeut le concept de «Cité muséale»: le visiteur est 
invité à circuler dans le musée comme dans une ville. Il dote le bâtiment d'un plan triangulaire, en écho à celui de la presqu'île, et suggère, par un découpage en trois sections, les trois missions essentielles dévolues au MAPA, inauguré en 1995 :

- un espace de présentation des collections permanentes, sur une superficie de $2700 \mathrm{~m}^{2}$, propose un parcours chronologique émaillé de présentations thématiques concernant la protohistoire et l'époque romaine ;

- une aile culturelle destinée à l'accueil des publics regroupe des ateliers, un auditorium et une bibliothèque spécialisée, créée à partir du fonds du Musée Réattu. Enrichie par le don de la bibliothèque de M. Jacques Coupry (professeur d'université, ancien directeur des Antiquités de la région Aquitaine et longtemps responsable des fouilles sur le site d'Olbia), elle recèle un fonds spécialisé en archéologie classique et histoire ancienne, privilégiant la période romaine, ainsi que le fonds iconographique du musée ;

- une aile scientifique est consacrée à la recherche archéologique et au stockage du mobilier archéologique. Le musée sert en effet de dépôt de fouilles. Des salles adaptées permettent un cheminement logique du mobilier, depuis son déchargement, son lavage, son triage, son étude, jusqu'à la présentation dans une vitrine du musée. Le laboratoire d'archéologie supervise une grande partie des fouilles programmées et surtout les fouilles de sauvetage sur le territoire urbain d'Arles. Il participe également au montage d'expositions temporaires, qui permettent de faire le point sur l'actualité des découvertes archéologiques à Arles. L'aile scientifique abrite en outre, depuis 1992, un Atelier de conservation et de restauration de mosaïques, le seul qui existe à ce jour en France, avec celui de Saint-Romain-en-Gal. Il a en charge les pavements exposés dans le Musée, entreposés dans ses réserves ou conservés in situ dans les cryptes et chantiers archéologiques arlésiens. Les nombreuses céramiques découvertes à Arles y sont également traitées et restaurées. L'Atelier participe aussi à la protection de pavements de différents musées et sites de l'ensemble des régions voisines. Il intervient ponctuellement, dans le cadre des missions archéologiques françaises à l'étranger, pour conserver les pavements mis au jour ou pour former les équipes locales à la conservation des mosaïques. Ces opérations permettent d'établir des contacts avec les collègues des différentes rives de la Méditerranée, mais aussi avec les divers organismes en charge du patrimoine culturel antique. Une telle coopération amène à recevoir au sein de l'atelier des stagiaires, étudiants en conservation ou restaurateurs venus expérimenter les techniques mises en œuvre à Arles.

\section{Les missions et les activités du Service des publics du MAPA}

$8 \quad$ Le Service des publics est chargé de définir et de mettre en œuvre une politique globale d'accueil des publics, au-delà des seuls groupes scolaires. Il joue un triple rôle au sein du musée.

1. Il conçoit et met en œuvre un programme d'activités donnant aux publics jeune et adulte de multiples accès aux collections permanentes et expositions temporaires (visites générales et thématiques, ateliers, etc.). Ce rôle de médiation est complété par l'élaboration d'une gamme d'outils d'aide à la visite mobilisant divers supports (livrets, parcours, multimédia).

2. Il participe à la valorisation des activités scientifiques du musée en proposant des 
rencontres avec les conservateurs, les restaurateurs et les archéologues. Des visites des espaces dédiés aux activités de recherche du musée sont organisées régulièrement, ainsi que des « randonnées archéologiques » permettant de faire connaître des sites de la région. C'est dans le cadre de cette politique de diffusion scientifique que le Service des publics a collaboré avec le pectorat d'Aix-Marseille, afin de concevoir un CD-Rom destiné à sensibiliser les jeunes aux multiples facettes du monde de l'archéologie : La machination de l'empereur. Une enquête de l'Agence des Aventuriers Archéologues. L'acquisition des connaissances se fait par le biais d'une enquête ludique sur la disparition mystérieuse d'objets conservés dans le MAPA.

3. Il sert enfin de relais avec l'extérieur du musée, puisqu'il inscrit son action dans le territoire départemental en liaison avec de multiples opérateurs (sociaux, culturels et touristiques) et en prise directe avec les ressources archéologiques des Bouches-duRhône. Des partenariats permettent de programmer des activités autour de la musique, du théâtre, du chant, de la danse... Le Service des publics prend ainsi appui sur l'actualité locale et nationale pour organiser des manifestations autour du MAPA, afin d'attirer le public jusque dans le musée. Les journées romaines d'Arles, baptisées Arelate $^{2}$, qui ont lieu chaque été au mois d'août, constituent l'une de ces occasions. Elles se sont associées au Festival du film péplum, créé il y a vingt ans.

9 Le musée organise aussi depuis six ans, en liaison avec le Rectorat de l'Académie AixMarseille, le Forum « Archéologie au collège » qui s'efforce de promouvoir l'archéologie en milieu éducatif. La dernière édition, qui s'est tenue le 15 mai 2007, a offert l'occasion à plusieurs classes de collégiens de présenter les projets Patrimoine et Archéologie qu'ils ont menés avec leurs enseignants en 2006-2007 ${ }^{3}$.

10 Parce que le Service a le souci de mieux connaître les attentes du public et les représentations qu'il se fait de l'Antiquité, il a mis sur pied deux dispositifs d'enquête. Le premier, de grande envergure, a été entrepris en partenariat avec l'office du tourisme d'Arles et vise à évaluer la façon dont les visiteurs appréhendent la dimension patrimoniale de la ville. Le second, d'une ampleur plus limitée, concerne les participants du festival Arelate; il s'agit de déterminer l'impact de l'archéologie expérimentale auprès du public.

11 Le Service des publics a également la volonté de tisser des liens avec d'autres parties de la Méditerranée et a établi des contacts avec le Centre d'Études Alexandrines, dirigé par Jean-Yves Empereur. Ainsi est né un projet de collaboration entre les élèves d'un collège arlésien et ceux de deux écoles d'Alexandrie, autour de l'élaboration d'un webcartoon. L'objectif était d'amener les enfants à réfléchir sur le patrimoine archéologique de leur ville, mais aussi de leur faire découvrir le patrimoine de la Méditerranée. Ce webcartoon retrace l'histoire d'un archéologue français qui découvre dans le Rhône l'épave d'un navire romain et contacte une collègue à Alexandrie. Ensemble, ils tentent de reconstituer le périple de ce bateau à travers la Méditerranée $\mathrm{du} \mathrm{I}^{\mathrm{er}}$ siècle de notre ère. Il sera présenté au public en 2008, dans le cadre d'une exposition programmée par le MAPA autour du thème "Mare Nostrum ». Celle-ci permettra de faire le point sur les récentes découvertes archéologiques qui ont été effectuées dans le Rhône et qui attestent qu'Arelate était un port dynamique, largement ouvert sur la mer Méditerranée. 


\section{Une façon originale de découvrir l'Antiquité, au miroir de l'œuvre d'Ingres}

Le MAPA s'est associé au Musée Ingres de Montauban pour co-organiser une exposition intitulée "Ingres et l'Antique ». Elle a d'abord été présentée à Montauban, du 15 juin au 15 septembre 2006, puis a été accueillie à Arles, du 2 octobre 2006 au 2 janvier 2007. Le partenariat établi entre les deux musées a permis d'allier la richesse du fonds documentaire de l'un à la compétence archéologique de l'autre. Cette initiative neuve, consistant à croiser l'histoire de l'art et l'archéologie, a pris appui sur une recherche universitaire menée il y a dix ans par Pascale Picard-Cajan, actuellement Conservateur du MAPA. Sa thèse de doctorat portait sur l'étude d'une série inédite de calques et de dessins ayant appartenu à Ingres et reproduisant des éléments de décors de vases grecs ${ }^{4}$. Ils avaient été réalisés par le peintre à partir des originaux ou de publications archéologiques. Les analyses de Pascale Picard-Cajan ont permis de préciser les rapports entretenus par l'artiste avec le milieu de l'archéologie, mais aussi de souligner le rôle joué par le patrimoine antique dans le laboratoire de son activité créatrice. La décision récente $\mathrm{du}$ musée de Montauban d'entreprendre une campagne de restauration de la collection de céramiques d'Ingres a fourni l'occasion de donner davantage d'ampleur et d'écho à cette enquête.

L'exposition, riche de plus de cinq cents pièces, présentait ainsi l'intérêt de mettre en regard une collection relevant habituellement des Beaux-Arts avec une collection d'archéologie - une approche interdisciplinaire réussie, qui a permis d'attirer de nouveaux publics (environ 50000 visiteurs au total). S'y côtoyaient des œuvres d'Ingres (peintures, dessins et relevés graphiques), des pièces archéologiques issues de sa collection personnelle ou de grandes collections européennes (vases grecs, marbres et terres cuites), ainsi que des volumes de publications scientifiques du $\mathrm{xIX}^{\mathrm{e}}$ siècle, connues et consultées par le peintre. Un parcours fort bien conçu, organisé autour de quatre grands thèmes ("Apprendre l'Antique", "Capturer l'Antique", "Métamorphoses ", «L'invisible héritage »), invitait le visiteur à suivre les différentes étapes du processus créatif, depuis la quête archéologique motivée par la recherche du Beau, source d'inspiration, jusqu'à l'œuvre finie, en passant par les différentes phases de gestation et de réélaboration. Les dispositifs muséographiques visaient à susciter un dialogue dynamique entre les artefacts antiques et les productions du peintre. Ainsi, le tableau intitulé Les Ambassadeurs d'Agamemnon, qui permet à Ingres d'obtenir en 1801 le premier prix de Rome et lui confère le titre de " peintre d'Histoire ", était exposé à côté du moulage du prototype ayant servi à représenter le personnage d'Ulysse - le "Phocion du Vatican", une copie romaine d'une statue grecque du ve siècle avant notre ère. On sait qu'Ingres avait eu l'occasion de l'observer au musée du Vatican, lorsqu'il était à Rome, et qu'il avait pris soin d'en faire pour lui-même un dessin, avant d'en expédier un moulage à Paris.

Un réel effort a été fait pour favoriser l'interactivité et solliciter la participation du public. Un ingénieux système de meubles à tiroirs permettait notamment au visiteur de découvrir, au gré de sa curiosité, quelques-unes des deux cents planches qui constituaient la documentation graphique de l'artiste, en les confrontant à une série d'originaux (ouvrages gravés, sculptures, vases). Autre atout de l'exposition: la présentation d'une maquette inédite, confectionnée par Isabelle Foriel Destezet, et destinée à proposer une reconstitution d'un temple grec miniature exécuté en 1856 par 
un ami d'Ingres, l'architecte Hittorff, à la demande du prince Louis-Napoléon. L'artiste avait réalisé la peinture située sous le portique arrière de l'édifice (La naissance de la dernière Muse). De l'œuvre, malheureusement disparue durant la Commune, il ne restait à ce jour que quelques photographies, ainsi que les dessins aquarellés de Hittorff.

$\mathrm{Au}$ fil de son cheminement dans les méandres du laboratoire imaginaire d'Ingres, le visiteur a ainsi pu découvrir les goûts esthétiques de l'artiste, en particulier sa prédilection pour ce que l'on appelle encore à l'époque les "vases étrusques». Les canons de la sculpture grecque (dont le modèle est fourni par la frise du Parthénon) et, plus encore, le tracé expert des peintres céramistes fournissaient à ses yeux la meilleur source d'inspiration en matière de dessin, de contours et de formes. L'exposition a bien montré comment le peintre, tel un aède, re-composait, en assemblant divers éléments inspirés de l'art antique. Le tableau Jupiter et Thétis (1811), dont le thème est puisé chez Homère, une des lectures favorites de l'artiste, constitue un excellent témoignage de la façon dont s'effectuait la réappropriation de l'héritage grec. La figure de Thétis est tirée d'un décor de vase campanien de la seconde collection Hamilton ; le piédestal du trône est décoré d'une gigantomachie issue d'un motif d'un camée hellénistique, conservé au musée de Naples; le visage intransigeant de Zeus reprend l'iconographie traditionnelle des statues du dieu Sérapis. C'est dans l'assemblage et la mise en scène de ces éléments hétéroclites que réside le génie créateur d'Ingres. Celui-ci concevait son activité comme une quête inlassable de la perfection du "trait grec »; il la parachevait par l'ajout des couleurs, seul domaine dans lequel les Modernes pensaient avoir surpassé les Anciens le grand Winckelmann lui-même le reconnaissait.

L'exposition a fait l'objet d'une mise en valeur originale et innovante. Outre la parution du catalogue, qui recèle une série d'articles scientifiques explorant les divers aspects du rapport qu'Ingres entretenait avec l'art antique ${ }^{5}$, un livret pédagogique a été édité en partenariat avec le CRDP d'Aix-Marseille ${ }^{6}$. Il renferme un CD-Rom qui propose une « archéologie » de deux œuvres: Stratonice ou la maladie d'Antiochus (1834) et La naissance de la dernière Muse (1856). On peut notamment y suivre les différentes phases de la confection de la copie du temple miniature de Hittorff. Enfin, pour accompagner et prolonger l'exposition, le service éducatif du Musée a mis en place une programmation spécifique destinée aux scolaires. De nombreux enfants et adolescents ont ainsi pris part à des ateliers de dessin. Leurs productions graphiques ont été mises à l'honneur dans le cadre d'une seconde exposition. Intitulée "Sur les traces d'Ingres ", elle a eu lieu du 14 mai au 24 juin 2007 et a permis d'envisager sous un prisme nouveau la passion d'Ingres pour l'Antique.

\section{ANNEXES}

\section{Contact :}

Musée de l'Arles et de la Provence antiques

Presqu'île du Cirque Romain, BP 205

13635 Arles cedex 
Tél. $0490188888 \cdot$ Fax 0490188893

info.mapa@cg13.fr

Le site du MAPA : www.arles-antique.cg13.fr (très complet et fort bien conçu, il propose des informations sur les activités et les événements, un aperçu des collections et de la muséographie, une présentation générale du musée, ainsi que des ressources pédagogiques et documentaires).

\section{NOTES}

1. Le Musée va mettre en valeur une partie de ce vestige exceptionnel, très rare en France : en 2010 seront aménagés un potager antique et un hippodrome végétal.

2. Arelate est le nom de la colonie fondée par Jules César en 46 avant notre ère.

3. Cf. le site de l'association ArchéomED :

http://archeomed.blog4ever.com/blog/article-73658.html.

4. P. PICARD-CAJAN, Ingres et l'antique: Étude d'un fonds documentaire consacré à la céramique grecque, thèse de doctorat dirigée par A.-F. Laurens, Montpellier, Université Paul Valéry, 1995.

5. P. PICARD-CAJAN (dir.), Ingres et l'antique : l'illusion grecque, Arles, Actes Sud, 2006.

6. P. PICARD-CAJAN (dir.), Ingres et l'Antique. Le laboratoire secret, Arles, Édition MAPA-CRDP AixMarseille, 2006. 